\title{
Super-resolution Reconstruction of Noisy Video Image Based on Sparse Representation Algorithm
}

\author{
Tierui Zhang, Dandan Li, Yanxia Cai and Yanyan Xu \\ Hengshui University, Hengshui, Hebei 053000, China \\ E-mail: ddli_dan@yeah.net
}

Keywords: sparse representation, super-resolution, image reconstruction, image denoising, video image, image processing

Received: July 29, 2019

\begin{abstract}
In this paper, the image super-resolution reconstruction (SRR) based on sparse representation was studied. Firstly, the sparse representation algorithm was simply analyzed, and then applied to the SRR processing of single image. In noisy video images, the Lucy-Rechardson algorithm was used for denoising first, then Lucas Kanade + multi-scale autoconvolution (MSA) method was used to register video images, and finally SRR was processed by sparse representation algorithm. Three video images were taken as examples for analysis, and the peak signal to noise ratio (PSNR) value and the structural similarity index measurement (SSIM) value were used as image quality evaluation indexes. The results showed that the average PSNR value and average SSIM of the SRR processing method based on sparse representation were significantly higher than those of bicubic interpolation method; the quality of the processed image was higher and the super-resolution effect was better. The experimental results prove the reliability of the proposed method and make some contributions to the further application of the sparse representation algorithm in SRR processing.
\end{abstract}

Povzetek: Predstavljena je metoda za rekonstrukcijo kvalitetne slike iz slabih posnetkov s kombiniranjem vrste algoritmov.

\section{Introduction}

In general, the higher the resolution of an image, the clearer the image and the stronger the ability to express details. After a certain imaging process for highresolution (HR) scenes, low-resolution (LR) images are obtained due to degradation processes such as blurring and noise, but LR images are required in many applications. At present, the commonly used methods to improve image quality include image denoising, restoration, enhancement, and image super-resolution reconstruction (SRR). SRR refers to a method of reconstructing an HR image through one or more LR images [1]. SRR technology is an ill-posed inverse problem [2, 3], which can acquire LR images without changing the hardware conditions, and it is of great value in the field of image processing. Sparse representation algorithms are also widely used in SRR processing [4].

In this paper, the application of sparse representation algorithm in SRR processing was studied, and a SRR processing method based on sparse representation of noisy video images was designed. The effectiveness of the proposed method was proved by an example analysis, which was beneficial to the better application of sparse representation algorithm in SRR processing and also provided some theoretical support for SRR processing of noisy video images.

\section{Related works}

Xing et al. [5] designed a novel neural network with barycentric weight function (BWFNN) method, and reconstructed image details through nonlinear center-ofgravity weight functions, showing excellent efficiency in image reconstruction. Dai et al. [6] proposed an improved projections onto convex sets (POCS) method to obtain the initial estimation of HR images by iterative curvaturebased interpolation (ICBI). The experimental results of subjective evaluation and objective evaluation proved the effectiveness of this method. Chen et al. [7] designed a regularization model based on the anisotropic fractional order adaptive (AFOA) specification, applied it to SRR image processing, and found that the model could achieve adaptive removal of image noise and well protect image edges. The experimental results showed that the image quality obtained by this method was good. Wang et al. [8] created a series of nested neighborhoods to collect LR pixels and then estimate the HR pixel values. This is a non-iterative method, which does not encounter convergence problems, but also has high computational efficiency.

\section{Sparse representation algorithm}

\subsection{Sparse representation}

The sparse representation of signal can be expressed as: $\min \|a\|_{0}$, st. $x=D a$, where $a$ is sparse representation coefficient, $D$ is over-complete dictionary, and \|\|$_{0}$ is the number of non-zero elements of a vector. Assuming that the limiting error must within $\sigma$, the sparse 
representation problem can be written as: $\min \|a\|_{0}, s t .\|x-D a\|_{2}<\sigma$.

\subsection{Sparse coding}

The method to solve the sparse representation problem is sparse coding, also known as sparse decomposition, which can be expressed as: $\tilde{a}=\underset{a}{\arg \min }\|a\|_{0}$, st.Da $\approx x \quad$ In this paper, the orthogonal matching pursuit (OMP) algorithm is chosen to solve this problem.

The original signal is expressed as $y$, and the given sparsity is $\mathrm{k}$; initial margin $r_{0}=y$, supporting index set $I_{0}=\phi$, and initial iteration number $k=1$. The supporting index is calculated in the $k$-th cycle: $\lambda_{k}=\underset{i=1,2, \cdots, N}{\arg \min }\left|\left\langle r_{k-1}, d_{i}\right\rangle\right|$, then the support set is $I_{k}=I_{k-1} \cup \lambda_{k}$. The residual is updated: $r_{k}=y-D_{\lambda_{k}}\left(D_{\lambda_{k}}^{T} D_{\lambda_{k}}\right)^{-1} D_{\lambda_{k}}^{T} y$. After loop iteration for $k$ times, when $\|y-D a\|<\sigma$, the sparse coefficient $a=D_{\lambda_{k}}\left(D_{\lambda_{k}}^{T} D_{\lambda_{k}}\right)^{-1} D_{\lambda_{k}}^{T} y$ is output.

\section{SRR processing of noisy video images under sparse representation}

\subsection{SRR processing of single image}

It is assumed that the sparse coefficients of image blocks are the same under HR dictionary $D_{h}$ and LR dictionary $D_{l}$. In SRR processing, the HR image is firstly degraded to LR image, and the training sample pair composed of HR image block and LR image block is obtained. After training $D_{h}$ and $D_{l}$, the sparse coefficient $a_{\text {of LR image }}$ block ${ }^{x_{l}}$ is calculated on $D_{l}$, and the reconstructed HR image block is obtained through $x_{h}=D_{h} a$.

The mapping relationship between HR image block and LR image block is represented by a sparse dictionary, and the single dictionary training model is established as follows:

$$
D=\underset{D \Lambda}{\arg \min }\|X-D \Lambda\|_{2}^{2}+\lambda\|\Lambda\|_{1}, s t .\left\|d_{i}\right\|_{2}^{2} \leq 1, i=1,2, \cdots N
$$

where $X=\left\{x_{1}, x_{2}, \cdots, x_{M}\right\}$ represents an image block, $M$ represents the total number of samples, $\Lambda=\left\{a_{1}, a_{2}, \cdots, a_{M}\right\}$ represents a sparse coefficient matrix, $N$ represents the number of dictionary atoms, and $\lambda$ is a Lagrangian multiplier used for balancing fidelity and sparsity.
In the image SRR, the two dictionaries can be expressed as:

$$
\begin{aligned}
& D_{h}=\underset{D_{h} \Lambda}{\arg \min }\left\|X_{h}-D_{h} \Lambda\right\|_{2}^{2}+\lambda\|\Lambda\|_{1}, \\
& D_{l}=\underset{D_{l} \Lambda}{\arg \min }\left\|Y_{l}-D_{l} \Lambda\right\|_{2}^{2}+\lambda\|\Lambda\|_{1},
\end{aligned}
$$

where $X_{h}$ and $Y_{l}$ are training sample matrices composed of HR image blocks and LR image blocks respectively.

The two dictionaries are trained jointly and expressed as:

$$
\min _{\left\{D_{h}, D_{l}, \Lambda\right\}} \frac{1}{P}\left\|X_{h}-D_{h} \Lambda\right\|_{2}^{2}+\frac{1}{Q}\left\|Y_{l}-D_{l} \Lambda\right\|_{2}^{2}+\lambda\left(\frac{1}{P}+\frac{1}{Q}\right)\|\Lambda\|_{1}
$$

where $P$ and $Q$ represent the sample number of HR and LR image blocks respectively.

The training model of the dictionary can be expressed as:

$$
\min _{\{\bar{D}, \Lambda\}}\|\bar{X}-\bar{D} \Lambda\|_{2}^{2}+\bar{\lambda}\|\Lambda\|_{1}, \bar{X}=\left[\begin{array}{l}
\frac{1}{\sqrt{P}} X_{h} \\
\frac{1}{\sqrt{Q}} Y_{l}
\end{array}\right], \bar{D}=\left[\begin{array}{l}
\frac{1}{\sqrt{P}} D_{h} \\
\frac{1}{\sqrt{Q}} D_{l}
\end{array}\right]
$$

\subsection{Video image SRR processing}

SRR was performed on video images on the basis of SRR for single image. The process was mainly divided into two steps: (1) registering LR sequence images; (2) obtaining HR image by SRR processing of image.

\subsubsection{Motion estimation}

In this paper, a hybrid non-display motion estimation method combining Lucas Kanade and multi-scale autoconvolution (MSA) was used to achieve image registration. Firstly, the initial motion of the image block was estimated by MSA, and then Lucas Kanade method was used for further registration to achieve accurate calculation of motion displacement. The steps of the hybrid method are as follows.

The current frame image is represented by $f$, the previous frame is represented by $f+1$, and the next frame is represented by $f-1$. The image $f$ is divided into blocks with the size of $m_{1} \times n_{1}$, and the position of the current frame image block $R$ is expressed as $(\Delta x, \Delta y)$. The position $\left(\Delta x_{1}, \Delta y_{1}\right)$ of the image blocks corresponding to the front and rear frames was calculated by MSA method, and the search range is $s_{1} \times s_{2}$, then the average motion displacement $\left(\Delta x_{2}, \Delta y_{2}\right)$ of the image blocks of three frames was calculated by using the motion displacement $\left(\Delta x_{1}, \Delta y_{1}\right)$. The image block $M$ of $m_{2} \times n_{2}$ is intercepted in $R$, and the corresponding image blocks $M^{\prime}$ and $M^{\prime \prime}$ before and after frames are intercepted by motion displacement. The motion displacement of the image blocks $M^{\prime}$ and $M^{\prime \prime}$ 
matched with $M$ can be expressed as $\left(\Delta x_{1}, \Delta y_{1}\right)+\left(\Delta x_{2}, \Delta y_{2}\right)$.

\subsubsection{SRR processing}

LR video sequence is set as $\left\{\cdots, \tilde{I}_{k-1}, \tilde{I}_{k}, \tilde{I}_{k+1}, \cdots\right\}$, and upsampling video sequence as $\left\{\cdots, I_{k-1}, I_{k}, I_{k+1}, \cdots\right\}$. After registration, the image block of each frame is $\left(\cdots, x_{k-1}, x_{k}, x_{k+1}, \cdots\right)$, The trained dictionary $D_{l}$ and $D_{h}$ are used to solve the sparse problem, the block $x_{k}^{h}$ of the current frame after SRR is obtained, and the whole video sequence is restored.

\section{Image quality evaluation index}

It is assumed that a $M \times N$ noisy video image is $f(i, j)$, and the image processed by $\operatorname{SRR}$ is $\bar{f}(i, j)$. The indicators for evaluating the quality of the image processed by SRR are:

(1) Peak signal to noise ratio (PSNR):

$$
\begin{aligned}
& P S N R \\
& =10 \log _{10}\left\{\frac{255^{2}}{\frac{1}{M \times N} \sum_{i=1}^{M} \sum_{j=1}^{N}[f(i, j)-\bar{f}(i, j)]^{2}}\right\}
\end{aligned}
$$

the larger the PSNR value, the better the image quality.

(2) Structural similarity ratio (SSIM):

$S S I M=\frac{\left(2 \mu_{x} \mu_{y}+C_{1}\right)\left(2 \sigma_{x y}+C_{2}\right)}{\left(\mu_{x}^{2}+\mu_{y}^{2}+C_{1}\right)\left(\sigma_{x}^{2}+\sigma_{y}^{2}+C_{2}\right)}$, where $\mu_{x}$ and $\mu_{y}$ are the mean values of the two images, $\sigma_{x}$ and $\sigma_{y}$ represent the standard deviation, $\sigma_{x y}$ represents covariance, and $C_{1}$ and $C_{2}$ are small positive numbers with denominators of 0 or close to 0 [9]. The closer the SSIM value to 1 , the higher the similarity between the processed image and the original image, and the better the image quality.

\section{Case analysis}

\subsection{Noise video image preprocessing}

In this paper, three road monitoring video were taken as examples to analyze the performance of the SRR method designed in this paper, and randomly selected some frame from the video image, as shown in Figure 1.

As can be seen from Figure 1, the video had a low resolution and some noises. The noise in the video image needed to be removed before SRR is performed. In this paper, Lucy-Rechardson algorithm was used, and its iteration expression is:

$$
f^{(k+1)}=f^{(k)}\left[\left(\frac{g}{f^{(k)} \otimes h} \oplus h\right)\right],
$$

where $g$ is degraded images, $f$ is the estimation of the original undegraded image, $h$ is a known point

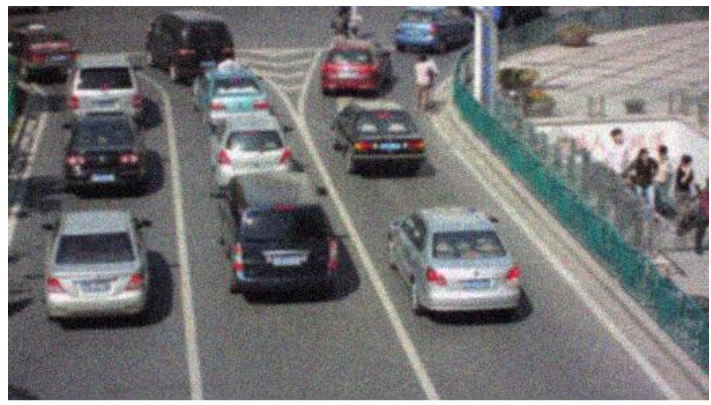

(1)

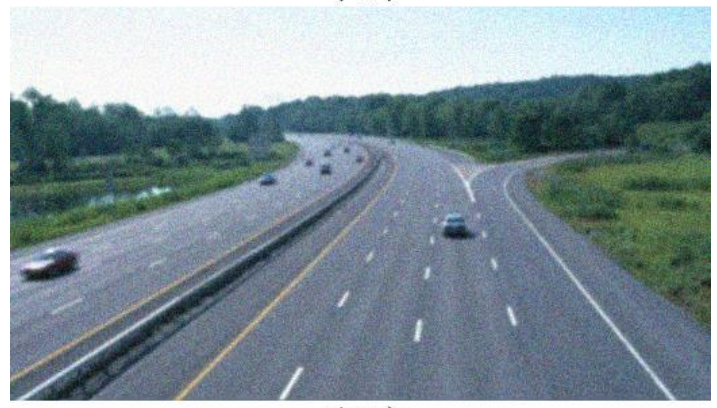

(2)

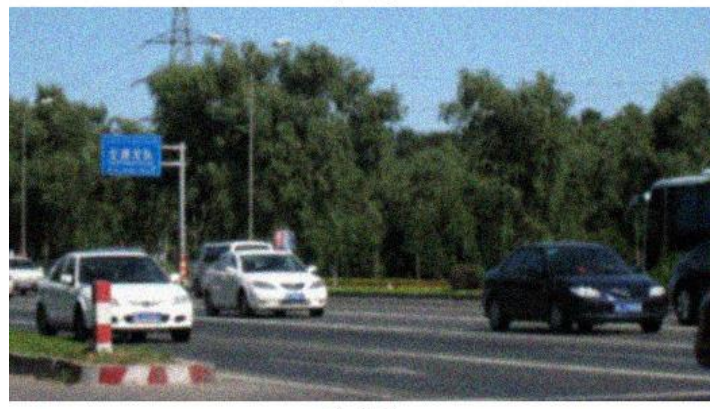

(3)

Figure 1: Noisy video images.

spread function, $\otimes$ is convolution, $\oplus$ is related operations, and $k$ is the number of iterations.

Lucy-Rechardson algorithm and wiener filter [10] are used for denoising, and the comparison results is shown in Figure 2.

Figure 3 is the local details of the image in Video 1, they are the original noisy image, the Lucy-Rechardson denoised image and the Wiener filtering denoised image, from left to right. Combined with Figure 2 and Figure 3, it can be found that the Lucy-Rechardson had better denoising effect than the Wiener filtering, which could better remove the noise in the image and retain the image details. 


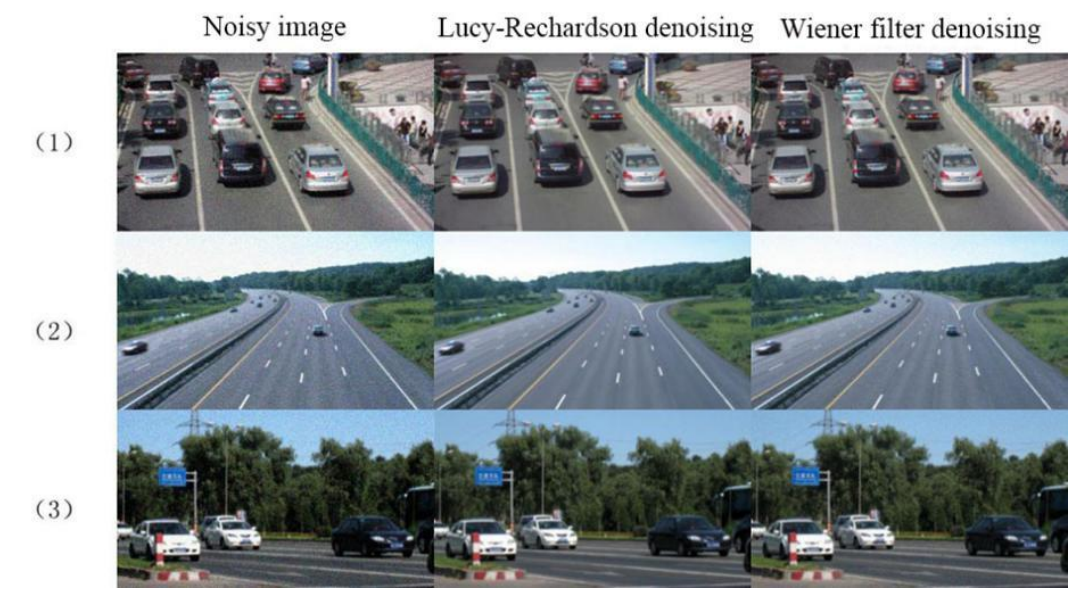

Figure 2: Comparison of denoising results.

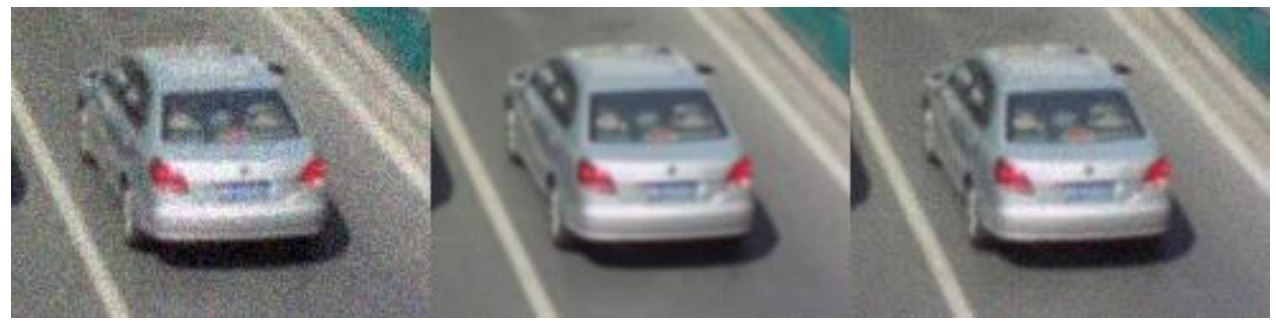

Figure 3: Local contrast

\subsection{SRR processing results of video image}

The HR and LR dictionaries were obtained by training the denoised video image sequence, then the image registration was realized by Lucas Kanade+MSA. The sparse coefficient was solved according to the registration image block, then the HR image blocks were restored by the obtained sparse coefficients, and the reconstructed HR video image sequences were obtained. The results are shown in Figure 4.

By comparing Figure 4 and Figure 1, it can be found that the quality of the video image after SRR processing was obviously improved, and the image details were more clear. To further understand the performance of the proposed method in this paper, it was compared with the bicubic interpolation method (a reconstruction method that obtains HR pixels from LR pixels by weighted averaging of the nearest sixteen sample points in a rectangular grid) [11] and ten consecutive frames of images in each video were taken. Video 1 was used as an example, and its PSNR value and SSIM value of video 1 are shown in Figure 5.

According to Figure 5 and 6, it can be found that the PSNR value of the video image reconstructed by this method was significantly higher than that of the bicubic interpolation method, and the SSIM value was closer to 1 , indicating that the reconstructed image had better super-resolution effect. The average values of the three video indicators were compared, and the results are shown in the Table 1.

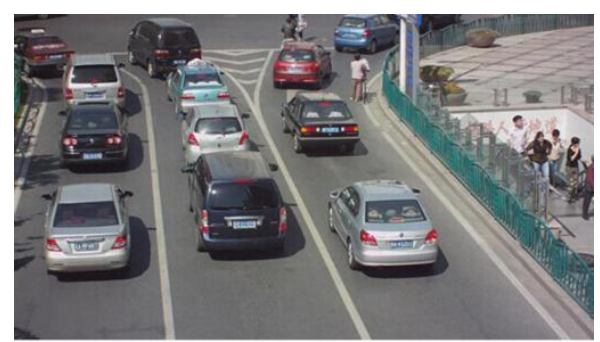

(1)

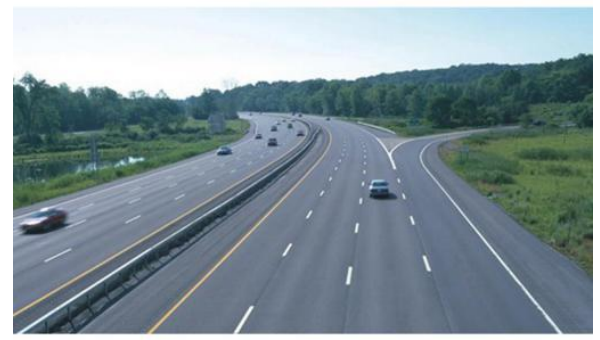

(2)

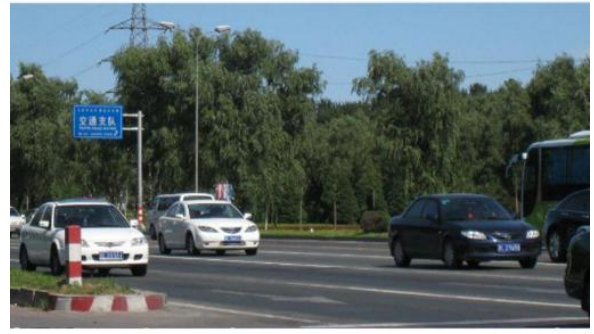

(3)

Figure 4: SRR results. 


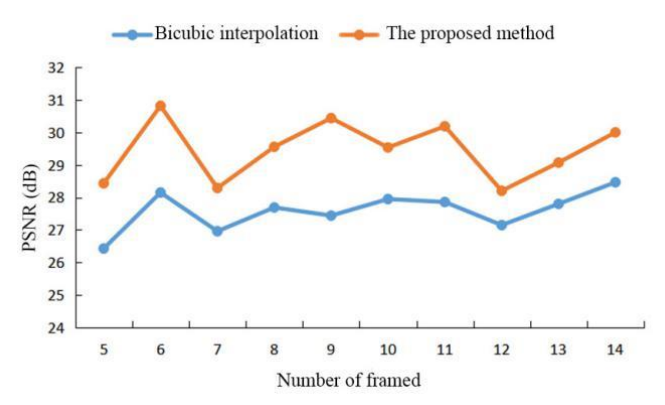

Figure 5: Comparison of PSNR.

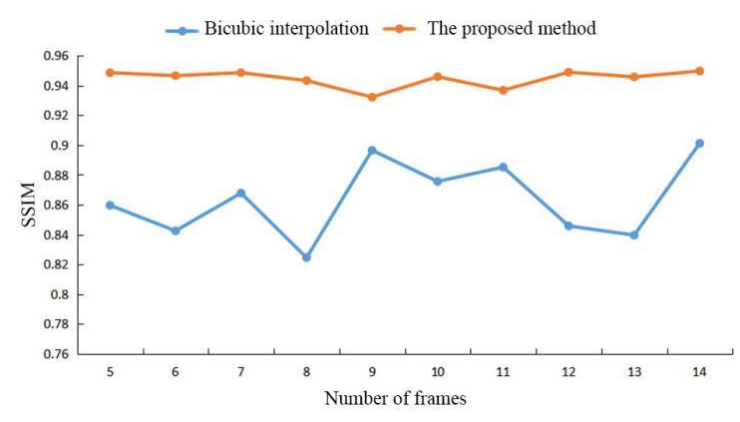

Figure 6: Comparison of SSIM

\begin{tabular}{|c|l|l|l|l|}
\hline \multicolumn{2}{|c|}{ Index } & Video 1 & Video 2 & Video 3 \\
\hline Average PSNR (dB) & Bicubic interpolation & 27.8557 & 26.4525 & 27.2154 \\
\cline { 2 - 5 } & Method of this paper & 29.4528 & 28.8745 & 29.4854 \\
\hline \multirow{2}{*}{ Average SSIM } & Bicubic interpolation & 0.8638 & 0.8542 & 0.8624 \\
\cline { 2 - 5 } & Method of this paper & 0.9445 & 0.9369 & 0.9486 \\
\hline
\end{tabular}

Table 1: Image quality evaluation results.

According to the results of Table 1, it can be found that in the three video images, the average PSNR value and the average SSIM value of the proposed method were higher than those of the bicubic interpolation. Taking Video 1 as an example, the average PSNR value of the image obtained by using the bicubic interpolation was $27.8557 \mathrm{~dB}$, and the average SSIM value obtained by this proposed method was $29.4528 \mathrm{~dB}$, which was obviously higher. The average SSIM value obtained by bicubic interpolation was 0.8638 , and that of proposed method was 0.9445 . According to the results of image quality evaluation, the SRR based on sparse representation designed in this paper had a higher super-resolution effect and image quality, which proved the reliability of the proposed method.

\section{Discussion}

SRR technology has important application value in many fields. In the aspect of entertainment, SRR technology can be used to recover low-resolution films and other influential materials from the 1980s to 1990s; in the aspect of medicine, it can improve the details of medical images such as Magnetic Resonance Imaging (MRI) [12], which can provide reliable basis for doctors' diagnosis [13]; in satellite remote sensing imaging, the LR image obtained by SRR is conducive to target recognition and provide reliable information for military investigation and environmental monitoring, etc. [14]. The application of SRR technology involves various fields of production and life, so it is of great practical significance to study SRR technology.

At present, the methods for image SRR processing mainly include interpolation-based algorithms, image sequence-based algorithms, maximum posterior probability methods, regularization methods [15] and so on. The sparse representation algorithm can reduce the amount of data in the calculation process, improve the quality of image reconstruction, and effectively avoid over-fitting and under-fitting, so it has a good application in SRR processing. This paper first designed a SRR processing method based on sparse representation for a single image and then carried out SRR processing for the noisy video image. In the video image, the LucyRechardson algorithm was firstly used to remove the noise in the video image, and then combined with Lucas Kanade algorithm and MSA algorithm to register the video image, and then SRR processing was carried out. According to the results of case analysis, it can be found that the SRR processing method based on sparse representation designed in this paper had high reliability. Firstly, from the results of image preprocessing, the denoising effect of the Lucy-Rechardson algorithm was better than that of Wiener filtering (Figure 2 and 3), which proved the reliability of the denoising algorithm in this paper. Then from the results of SRR processing, it can be seen from the comparison between Figure 1 and Figure 4 that, after SRR processing based on sparse representation designed in this paper, the resolution of the image was obviously improved, the image was clearer, and the details were more obvious. Taking PSNR and SSIM as image quality evaluation indexes, the method in this paper was compared with the bicubic interpolation method, and the results showed that the PSNR value and SSIM value of the method in this paper are both higher. In the three video images, the PSNR values of the images obtained by proposed method were $29.4528 \mathrm{~dB}, 28.8745$ $\mathrm{dB}$ and $29.48854 \mathrm{~dB}$, and the SSIM values were 0.9445 , 0.9369 and 0.9486 , respectively, which are significantly higher than that of the bicubic interpolation method. The results showed that the image obtained by SRR processing under the sparse representation designed in this paper had better super-resolution effect, which proves the reliability of this method.

SRR is an important part of image processing. Although some achievements have been obtained from the research on SRR processing under sparse representation algorithm in this paper, further research is needed, such as the research on denoising video image, the research on motion estimation method, the research on how to reduce the amount of SRR calculations, etc. 


\section{Conclusion}

Based on the sparse representation algorithm, the SRR processing of noisy video images was studied in this paper. Super-resolution reconstruction of image was carried out through sparse representation, and registration of video image was realized by Lucas Kanade+MSA hybrid algorithm. The results of the case analysis showed that the PSNR value and SSIM value of the reconstructed image obtained by the method designed in this paper were both higher, which proves the effectiveness of the sparse representation algorithm in SRR processing and is conducive to the further development of image SRR processing.

\section{Acknowledgement}

This study was supported by Research on the reform of theoretical teaching methods of Arts in Hengshui University-Taking the course of history of modern world design as an example under grant number jg2018085.

\section{References}

[1] Xuan, L., Wang, D., Shi, W., Deng, D.X. (2018). Group-based single image super-resolution with online dictionary learning. Geomatics \& Information Science of Wuhan University, 2016(1), pp. 84 . https://doi.org/10.1186/s13634-016-0380-9.

[2] Li, Y., Wang, Y., Li, Y., Jiao, L., Zhang, X., Stolkin, R. (2016). Single Image super-resolution reconstruction based on genetic algorithm and regularization prior model. Information Sciences An International Journal, 372(C), pp. 196-207. https://doi.org/10.1016/j.ins.2016.08.049.

[3] Zhao, S., Lyu, Z., Liang, H., Sarem, M. (2017). A mixed non-local prior model for image superresolution reconstruction. Chinese Journal of Electronics, 26(4), pp. 778-783.

https://doi.org/10.1049/cje.2016.06.024.

[4] Zhu, X., Jin, P., Wang, X.X., Ai, N. (2018). Multiframe image super-resolution reconstruction via low-rank fusion combined with sparse coding. Multimedia Tools \& Applications, pp. 1-12. https://doi.org/10.1007/s11042-018-6495-2.

[5] Huo, X., Tang, R.L., Ma, L.L., Shao, K., Yang, Y.H. (2018). A novel neural network for superresolution remote sensing image reconstruction. International Journal of Remote Sensing, pp. 1-11. https://doi.org/10.1080/01431161.2018.1516319.

[6] Dai, S., Cui, J., Zhang, D., Liu, Q., Zhang, X. (2017). Study on infrared image super-resolution reconstruction based on an improved POCS algorithm. Journal of Semiconductors, 38(4), pp. 78-82. https://doi.org/10.1088/1674-4926/38/4/044010.

[7] Chen, C., Liang, H., Zhao, S., Lyu, Z., Sarem, M. (2015). A novel multi-image super-resolution reconstruction method using anisotropic fractional order adaptive norm. Visual Computer, 31(9), pp. 1217-1231. https://doi.org/10.1007/s00371-014-1007-5.

[8] Wang, X., Zhou, D., Zeng, N., Yu, X., Hu, S. (2018). Super-resolution image reconstruction using surface fitting with hierarchical structure. Journal of Visual Communication and Image Representation, pp. S1047320318300592. https://doi.org/10.1016/j.jvcir.2018.03.011.

[9] Chen, Y., Hou, C.P., Zhou, Y. (2015). Image superresolution reconstruction based on improved neighbor embedding and structure self-similarity. Journal of Optoelectronics.laser, 26(8), pp. 16181625. https://doi.org/10.16136/j.joel.2015.08.0216.

[10] Ramanah, D.K., Lavaux, G., Wandelt, B.D. (2017). Wiener filter reloaded: fast signal reconstruction without preconditioning. Monthly Notices of the Royal Astronomical Society, 468(2), pp. 17821793. https://doi.org/10.1093/mnras/stx527.

[11] Qu, Y.S., Fan, X.W., Li, Y.C., Zhang, B.H. (2006). Multi-time wavelet bicubic interpolation search algorithm and its application to high-resolution reconstruction of remote sensing image. OptoElectronic Engineering, 33(5), pp. 62-41. https://doi.org/10.1360/crad20060325.

[12] Zhang, D., He, J., Zhao, Y., Du, M. (2015). MR image super-resolution reconstruction using sparse representation, nonlocal similarity and sparse derivative prior. Computers in Biology and Medicine, 58, pp. 130-145.

https://doi.org/10.1016/j.compbiomed.2014.12.023

[13] Hatvani, J., Horvath, A., Michetti, J., Basarab, A., Kouame, D., Gyongy, M. (2019). Deep learningbased super-resolution applied to dental computed tomography. IEEE Transactions on Radiation and Plasma Medical Sciences, 3(2), pp. 120-128. https://doi.org/10.1109/TRPMS.2018.2827239.

[14] Gou, S., Liu, S., Yang, S., Jiang, L.C. (2017). Remote sensing image super-resolution reconstruction based on nonlocal pairwise dictionaries and double regularization. IEEE Journal of Selected Topics in Applied Earth Observations \& Remote Sensing, 7(12), pp. 47844792. https://doi.org/10.1109/JSTARS.2014.2328596.

[15] Panda, S.S., Jena, G., Sahu, S.K. (2015). Image Super Resolution Reconstruction Using Iterative Adaptive Regularization Method and Genetic Algorithm. Indian Journal of Medical Research, 60(1), pp. 19-27. https://doi.org/10.1007/978-81-322-2208-8_62. 